\title{
Interactions of egg yolk lipoprotein fraction with boar spermatozoa assessed with a fluorescent membrane probe
}

\author{
Leyland Fraser, Lukasz Zasiadczyk, Jerzy Strzezek
}

\begin{abstract}
Warmia and Mazury University in Olsztyn, Department of Animal Biochemistry and Biotechnology, Olsztyn, Poland
\end{abstract}

\begin{abstract}
The interactions of a fluorescent membrane probe, 1-anilinonaphthalene-8-sulfonic acid (1,8-ANS), with boar spermatozoa were followed through the use of lipoprotein fraction of ostrich egg yolk (LPFo). Semen samples, extended in Kortowo 3 (K3) extender, were supplemented with $2 \%$ or $5 \%$ LPFo and stored for $3 \mathrm{~h}$ at $16^{\circ} \mathrm{C}$. Additionally, cold shocktreated spermatozoa $\left(1 \mathrm{~h}\right.$ at $\left.4^{\circ} \mathrm{C}\right)$ were stored in $\mathrm{K} 3$ extender supplemented with $\mathrm{LPFo}$ for $3 \mathrm{~h}$ at $16^{\circ} \mathrm{C}$. In each boar, the fluorescent enhancement of ANS was observed in K3-extended semen supplemented with LPFo, prior to storage. Following storage, there was a significant increase in LPFo-ANS fluorescence, particularly in the sperm membrane overlying the head and midpiece regions. There were significant differences among the boars with respect to the sperm populations defined by the LPFo-ANS fluorescence. Sperm viability was not significantly affected during the storage period. Furthermore, the proportions of spermatozoa defined by the different patterns of LPFo-ANS fluorescence were low and remained unchanged after storage of cold shock-treated spermatozoa with $2 \%$ or $5 \%$ LPFo, suggesting irreversible damage to the sperm membrane architecture. These findings indicate that the ANS fluorescent probe could be used to shed more light on the nature of the interactions between LPFo and sperm membrane following semen preservation. Such valuable information could contribute to the development of an optimal protocol for cryopreservation of boar semen.
\end{abstract}

Key words: boar, spermatozoa, lipoprotein fraction, ANS fluorescence

\section{Introduction}

Boar sperm membranes possess discrete domains of differing membrane fluidity, which is significantly affected by temperature [1-2]. Moreover, the sperm membranes appear to be the primary site of damage induced by reduced temperature and alterations in their molecular composition can impair the fertilization processes [3]. Evidence is assembled to suggest that lyophilized lipoprotein fraction of ostrich egg yolk (LPFo) has an overwhelming beneficial effect on the structural and functional integrity of boar plasma membrane during liquid storage [4-6] and cryopreservation of semen [7]. Recent studies in our laboratory have shown that artificial insemination of sows with liquid-stored or cryopreserved semen supplemented

Correspondence: L. Fraser, Dept. of Animal Biochemistry and Biotechnology, Warmia and Mazury University of Olsztyn, Oczapowskiego Str. 510-718 Olsztyn, Poland; tel. (+4889) 5233626, fax.: (+4889) 5240138, e-mail: fraser@uwm.edu.pl with LPFo gave acceptable fertility results [8-9]. There is abundant evidence indicating that the biochemical composition of LPFo is different from that of the lipoprotein fraction isolated from hen egg yolk, in terms of protein, cholesterol, phospholipids and fatty acid contents $[4-5,8]$. Little is known about the mechanism underlying the egg yolk lipoprotein interactions with sperm membrane architecture. However, many hypotheses have been suggested to explain the mechanisms by which lipoproteins of hen egg yolk protect spermatozoa during semen preservation [10-11].

The interactions of egg yolk lipoproteins with spermatozoa had been investigated using an enzymelabeled antibody method and radioactive labeling of egg yolk lipids [12]. Several authors demonstrated that a fluorescent membrane probe, 1-anilinonaphthalene8 -sulfonic acid (1,8-ANS), could be used to study the interactions of egg yolk components with ram or bovine spermatozoa [13-14]. It has been confirmed that ANS is hardly fluorescent in aqueous solutions, but becomes strongly fluorescent in apolar, organic solvents or when bound to hydrophobic molecules 
[15]. The fluorescent probe is generally recognized to bind membrane proteins and shows strong binding affinity for phospholipids, such as phosphatidylcholine and lysolecithin [16]. In this study, the membrane probe ANS was used to investigate the interactions of different concentrations of LPFo with boar spermatozoa following after a $3 \mathrm{~h}$-storage period at $16^{\circ} \mathrm{C}$. Additionally, the interactions of LPFo with cold shock-treated spermatozoa were studied before and after storage at $16^{\circ} \mathrm{C}$. It is envisaged that these experiments would provide an insight into the nature of the molecular interactions between LPFo and the sperm membrane following semen preservation. Sperm viability such as motility, plasma membrane integrity and mitochondrial function, was monitored before and after storage of $\mathrm{K} 3$-extended semen supplemented with $2 \%$ or $5 \%$ LPFo.

\section{Materials and methods}

Ejaculate collections. Semen was collected from 3 boars, designated F, K and M (aged 2 years, Polish Large White race) once weekly for a period of 3 weeks. The boars were fed with a commercial porcine ration. Water was available ad libitum. Semen was collected using the gloved-hand technique, and the gel portion was removed using nylon mesh filter. Sperm concentration was calculated with a hemocytometer. The percentage of morphologically abnormal spermatozoa was evaluated using the Giemsa staining method. Permission to conduct this study was granted by the Local Ethics Committee for Animal Experiments.

Semen processing and storage. Semen samples were divided into 2 parts: one part was used for liquid storage (Experiment 1) and the other was used for cold shock treatment (Experiment 2). The LPFo was isolated from ostrich egg yolk, lyophilized and stored until required. A brief description of the LPFo extraction procedure has been published elsewhere [4].

In Experiment 1, semen samples were extended $\left(30 \times 10^{6}\right.$ spermatozoa/ml) in $\mathrm{K} 3$ extender $(69.3 \mathrm{mM}$ fructose, $64.6 \mathrm{mM}$ sodium citrate, $8.0 \mathrm{mM} \mathrm{Na}$-EDTA, $14.2 \mathrm{mM}$ potassium acetate; $\mathrm{pH} 6.8$, $250 \mathrm{mg}$ gentamycin sulphate) supplemented with $2 \%$ and $5 \%(\mathrm{w} / \mathrm{v})$ LPFo. Samples $\left(5 \times 10^{6}\right.$ spermatozoa $\left./ \mathrm{ml}\right)$ taken for ANS fluorescent analysis prior to storage of K3-extended semen supplemented with $2 \%$ or $5 \%$ LPFo at room temperature were used as the control (0h). The extended semen was stored in a Thermobox for $3 \mathrm{~h}$ at $16^{\circ} \mathrm{C}$.

In Experiment 2, washed semen samples $(800 \times \mathrm{g}, 5 \mathrm{~min})$ were re-suspended in a phosphate-buffered solution (PBS) and subjected to cold shock treatment $\left(1 \mathrm{~h}\right.$ at $\left.4^{\circ} \mathrm{C}\right)$. Cold shocked-treated spermatozoa were rewashed $(800 \times \mathrm{g}, 5 \mathrm{~min})$, re-suspended in $\mathrm{K} 3$ extender supplemented with $2 \%$ and $5 \%(\mathrm{w} / \mathrm{v})$ and stored for $3 \mathrm{~h}$ at $16^{\circ} \mathrm{C}$.

Analysis of ANS fluorescence. The ANS analysis was performed according to a previous method [13], with some modifications. Sperm samples were washed $(800 \times \mathrm{g}, 5 \mathrm{~min})$ to remove unbound LPFo and re-suspended in PBS containing $1 \mathrm{mM}$ working solution of ANS (Molecular Probes, Eugene, OR, USA). Following incubation for $15 \mathrm{~min}$ at $37^{\circ} \mathrm{C}$, smears of ANS-treated sperm samples were made on clean microscopic slides, air-dried and examined under an epifluorescence microscope, equipped with appropriate excitation/emission filters (Olympus $\mathrm{CH}$ 30, Tokyo, Japan). In addition, washed spermatozoa $(800 \times \mathrm{g}, 5 \mathrm{~min})$, stored in the absence of LPFo for $3 \mathrm{~h}$ at $16^{\circ} \mathrm{C}$, were stained with ANS and analyzed, as described above. The degree of LPFo-ANS fluorescence was scored subjectively using the following scoring system for each group of spermatozoa: a - fluorescing simultaneously throughout the head and mid-piece regions; $\mathrm{b}$ - fluorescing only throughout the head region; c - fluorescing only throughout the mid-piece region; $\mathrm{d}$ - without any positive fluorescence in the head or midpiece region and $\mathrm{e}-$ stored in the absence of LPFo and stained with ANS. Slides were prepared in duplicate and approximately 200 spermatozoa were evaluated per slide. Spermatozoa showing intense, definite, weak or no fluorescence in their membrane structures were classified into 4 groups, a, b, c and d, as described above.

Sperm viability assessments. For sperm motility analysis aliquots of semen samples were placed on pre-warmed microscope slide, overlaid with a $20 \times 20 \mathrm{~mm}$ coverslide and examined at $200 \times$ magnification under a light microscope (Olympus BX 40, Tokyo, Japan), equipped with a heated stage $\left(37^{\circ} \mathrm{C}\right)$.

Sperma plasma membrane integrity (PMI) was assessed using the Live/Dead Sperm Viability Kit L-7011 (Molecular Probes, Eugene, OR, USA), consisting of a combination of fluorescent probes, SYBR-14 $(0.1 \mathrm{mg} / \mathrm{ml}$ dimethylsulfoxide, DMSO $)$ and propidium iodide, PI (2.4 $\mu \mathrm{M}$ PI in Tyrode's salt solution), as described in a previous study [17]. A working solution of SYBR-14 diluted (1:50) with HEPES buffered solution (10mM HEPES, $0.85 \% \mathrm{NaCl}$, $0.1 \%$ bovine serum albumin, $\mathrm{pH} 7.4$ ) was prepared. Briefly, diluted semen samples $\left(30 \times 10^{6} \mathrm{sperm} / \mathrm{ml}\right)$ were incubated with SYBR-14 and PI solutions for 10 minutes at $37^{\circ} \mathrm{C}$. Following incubtaion, aliqouts of stained sperm cells were placed on microscopic slides, covered with cover slips and examined at $600 \times$ magnification under an epifluorescence microscopy (Olympus $\mathrm{CH} 30$ ). A minimum of 200 cells per slide was examined in random fields of each aliquot and classified as intact or damaged plasma membrane.

The percentage of live spermatozoa with functional mitochondria was assessed using a combination of fluorescent stains, rhodamine 123 (R123, Molecular Probes, Eugene, OR, USA) and PI, as described in a previous study [7]. Rhodamine 123 was prepared from a stock solution of $5 \mathrm{mg} / \mathrm{ml}$ DMSO and stored in aliquots of $30 \mu \mathrm{l}$ until required. Aliquots $(10 \mu \mathrm{L})$ of samples stained with R123/PI were examined under an epifluorescence microscope (Olympus CH 30). Sperm cells displaying only green fluorescence at the mid-piece region were considered viable spermatozoa with functional mitochondria. A minimum of 200 cells per slide was examined in random fields of each aliquot.

Statistical analysis. All values were expressed as means \pm standard error of means (SEM). Data from Experiment 1 were analyzed by ANOVA using boar and sperm treatments (2\% and 5\% LPFo concentrations) as the main variables after a $3 \mathrm{~h}$-storage period. Differences between means were considered significant at $\mathrm{p}<0.05$, using the Neuman-Keuls post hoc test. The Statistica software package (StatSoft Incorporation, Tulsa OK., USA) was used to analyze the data.

\section{Results}

Fig. 1 shows the localization of fluorescence of ANS following its interactions with LPFo before or after storage. The interactions of LPFo with spermatozoa were accompanied by quite high ANS fluorescence intensity at different regions of the sperm membrane, as shown in Fig. 1 (groups a, b and c). There was weak ANS fluorescence for spermatozoa, which poorly interacted with LPFo (Fig. 1; group d). Furthermore, spermatozoa stored in the absence of LPFo exhibited very poor ANS fluorescence intensity, as shown in Fig. 1; group e. 

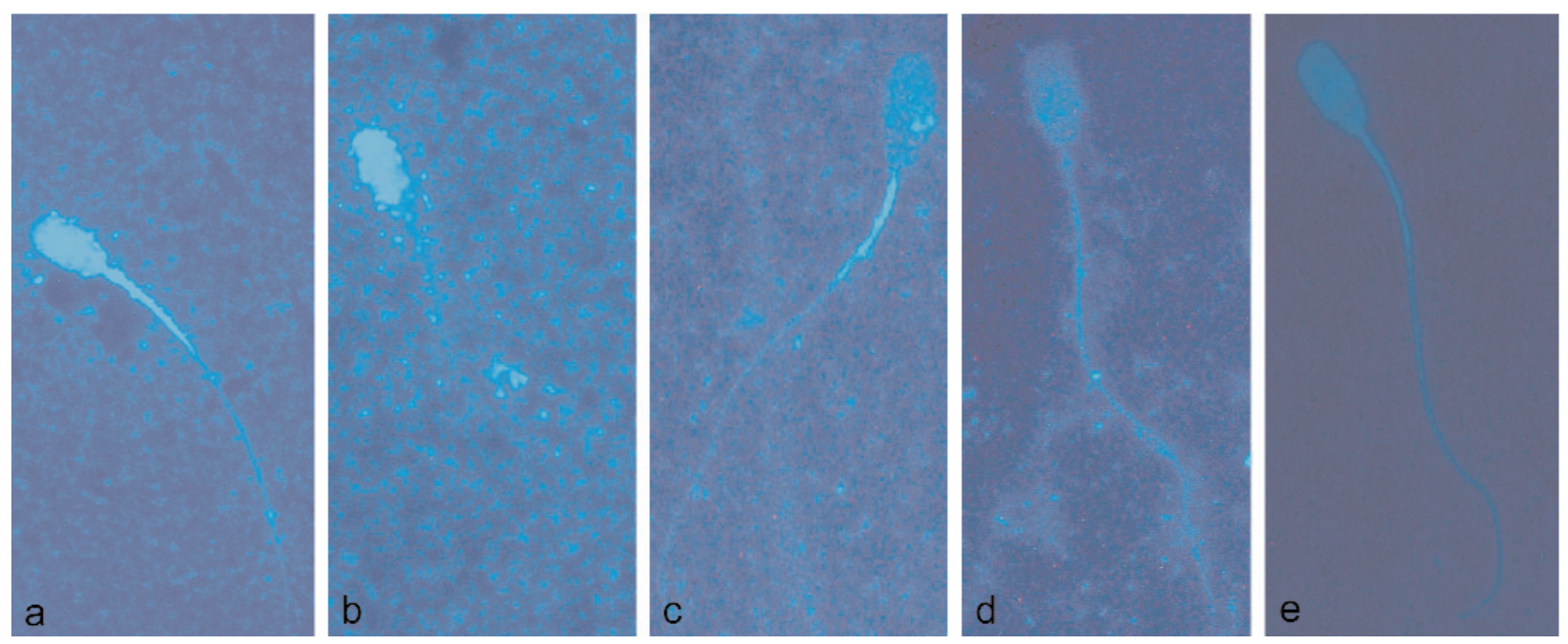

Fig. 1. Photographs of different patterns of LPFo-ANS fluorescence (original magnification $\times 400$ ) in boar spermatozoa. The LPFo-ANS fluorescence intensity was scored subjectively using the following scoring system for each group of spermatozoa: a. fluorescing simultaneously throughout the head and mid-piece regions; b. fluorescing only throughout the head region; c. fluorescing only throughout the mid-piece region; d. without any positive fluorescence in the head or midpiece region; e. stored in the absence of LPFo and stained with ANS.

In Experiment 1, ANOVA results revealed that only boar variability $(p<0.05)$ had a significant effect on LPFo-ANS fluorescence patterns in spermatozoa following storage at $16^{\circ} \mathrm{C}$. It was observed that treatment (2\% and 5\% LPFo concentrations) did not have any significant effect $(\mathrm{p}>0.05)$ on the different LPFo-ANS fluorescence patterns in the stored spermatozoa.

In Experiment 1, the proportions of spermatozoa defined by LPFo-ANS fluorescence over the head and midpiece regions (Fig. 2; group a) were markedly higher $(\mathrm{p}<0.05)$ compared to the other groups, regardless of LPFo concentration (Fig. 2). With progressive storage time, the LPFo-ANS fluorescence increased, spreading more evenly over the sperm head and midpiece. Consistent inter-boar variability was detected for the populations of spermatozoa defined by the LPFo-ANS fluorescence. Spermatozoa of Boar F depicted the highest percentage (approximately 1.5 to 2 -fold) of LPFo-ANS fluorescence over the head and midpiece regions compared with the other boars during storage in $\mathrm{K} 3$-extended semen supplemented with $2 \%$ LPFo (Fig. 2A) or 5\% LPFo (Fig. 2B).

In Experiment 2, the ANS fluorescence intensity showed that LPFo coating was not uniformly distributed on the membrane surface of cold-shocked spermatozoa and, in some cases, there was preferential binding of the lipoprotein fraction after storage. Spermatozoa showing LPFo-ANS fluorescence in the midpiece region (group b) or weak ANS fluorescence (group d) were predominant in $\mathrm{K} 3$-extended semen supplemented with $2 \%$ LPFo, irrespective of the storage period (Fig. 3A). In contrast, it was observed that a reduction in the proportions of spermatozoa showing LPFo-ANS fluorescence in the midpiece region (group b) was concomitant with an increase in the sperm pop- ulations showing weak ANS fluorescence (group d) after storage in K3-extended semen supplemented with 5\% LPFo (Fig. 3B).

There were no significant differences in sperm viability in terms of motility, PMI and mitochondrial function at $0 \mathrm{~h}$ and $3 \mathrm{~h}$ of storage at $16^{\circ} \mathrm{C}$, irrespective of the LPFo concentration. Overall, the percentage of motile spermatozoa averaged $67.5 \pm 2.2( \pm$ SEM). There were no marked differences ( $>0.05)$ in sperm motility among boars. However, sperm PMI and mitochondrial function differed among boars, being markedly lower in Boar $\mathrm{K}$ compared with Boar $\mathrm{F}$ and $\mathrm{M}$ during storage in $\mathrm{K} 3$-extended semen supplemented with $2 \%$ LPFo (Table 1).

\section{Discussion}

In this study, the hydrophobic fluorescence probe ANS was used to monitor the dynamic interactions between different concentration of LPFo and sperm membrane. It was observed that spermatozoa exposed to LPFo displayed a marked increase in ANS fluorescence, indicating the availability of fluorescence-active binding sites, particularly in the membrane overlying the head and midpiece regions. The overall distributions of ANS fluorescence became more apparent after a $3 \mathrm{~h}$ storage period, suggesting that coating of the sperm membranes by LPFo results in the exposure of multiple binding sites for the probe. Similar findings were reported by MacDonald and Foulkes [14], when ANS was used to study the interactions of bovine spermatozoa with seminal plasma or hen egg yolk lipoproteins. However, our results contrast the findings of Watson [13], which demonstrated that ANS fluorescence intensity was significantly reduced when ram sperma- 
A

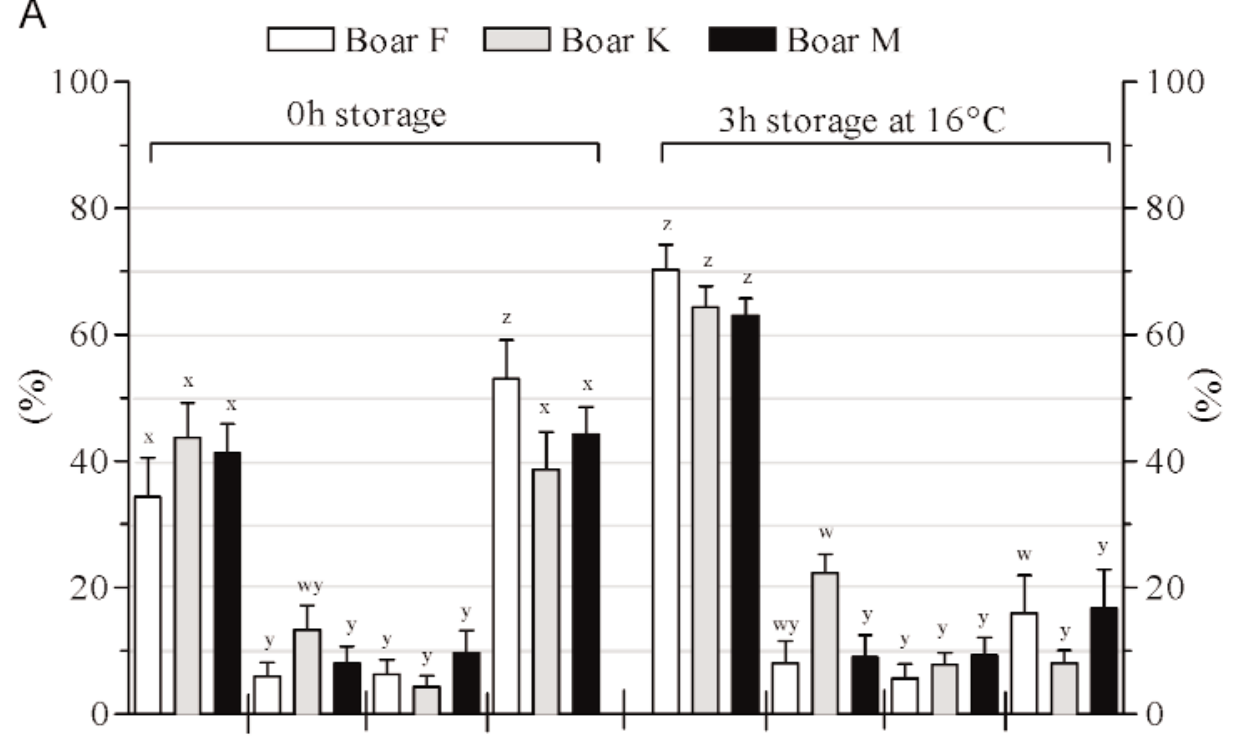

B

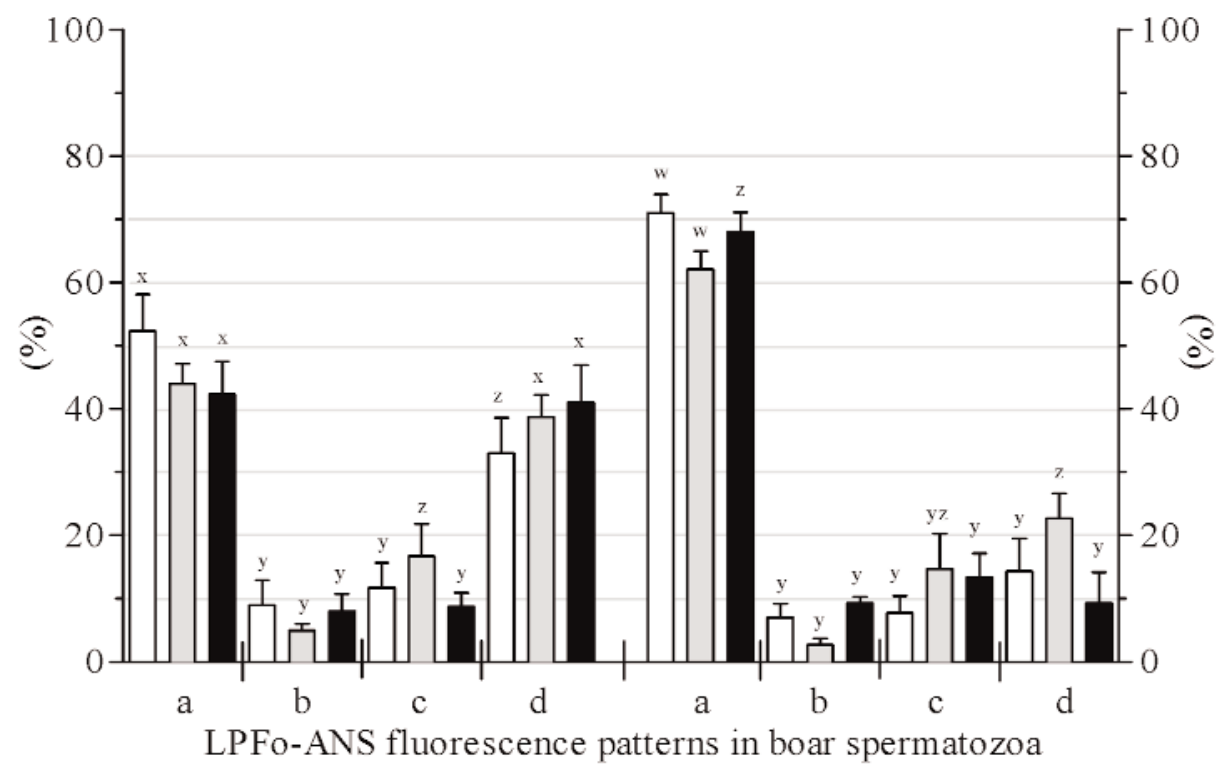

Fig. 2. Percentage of spermatozoa showing LPFo-ANS fluorescence patterns immediately after LPFo treatment $(0 \mathrm{~h})$ and after $3 \mathrm{~h}$ of storage at $16^{\circ} \mathrm{C}(3 \mathrm{~h})$. The $\mathrm{K} 3$-extended semen was supplemented with (A) $2 \%$ LPFo and (B) 5\% LPFo. The degree of LPFo-ANS fluorescence was scored subjectively using the following scoring system for each group of spermatozoa, as described in Fig. 1. Values represent the means $( \pm$ SEM) of 3 ejaculates, 3 each from the 3 boars. Within boars values with different letters $(\mathrm{w}, \mathrm{x}, \mathrm{y}, \mathrm{z})$ are significant at $\mathrm{p}<0.05$.

tozoa were incubated with different concentrations of whole hen egg yolk. In the present study, a quenching effect of either $2 \%$ or $5 \%$ LPFo on ANS fluorescence intensity of stored spermatozoa was not observed. This observation confirms that the interactions of spermatozoa with LPFo provided appropriate binding sites for the membrane probe ANS, particularly after the $3 \mathrm{~h}$ storage period.

It is noteworthy that ANS-related literature provides evidence that the hydrophobic ANS binds strongly to zwitterionic phospholipids of the membranes and does not give a fluorescence enhancement in the presence of anionic phospholipids because the negatively charged probe cannot penetrate into the hydrophobic regions of the bilayer [16,18]. It is, there- fore, possible that this assumption might explain the low binding affinity of ANS to spermatozoa, when stored in the absence of LPFo. It should be noted that the polarity of the surrounding environment is a major factor that could influence the ANS florescence [15]. Since ANS binding sites on LPFo structures constitute phospholipid and protein domains, it can be suggested that the large fluorescence response to electrostatic and/or hydrophobic changes in the environmental conditions was associated with increased binding affinity of the probe. Apparently, the ANS fluorescence enhancement displayed by spermatozoa stored with LPFo might be a reflection of higher quantum yield due to increased hydrophobicity of LPFo-ANS binding sites. Moreover, it has been demonstrated that 
A

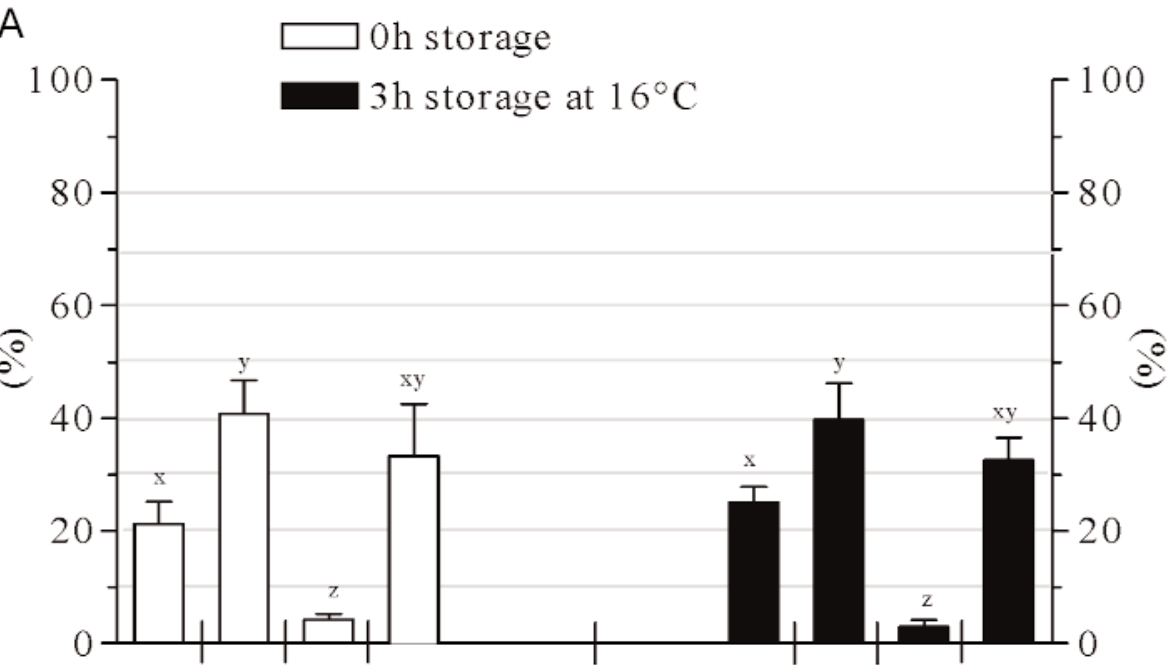

B

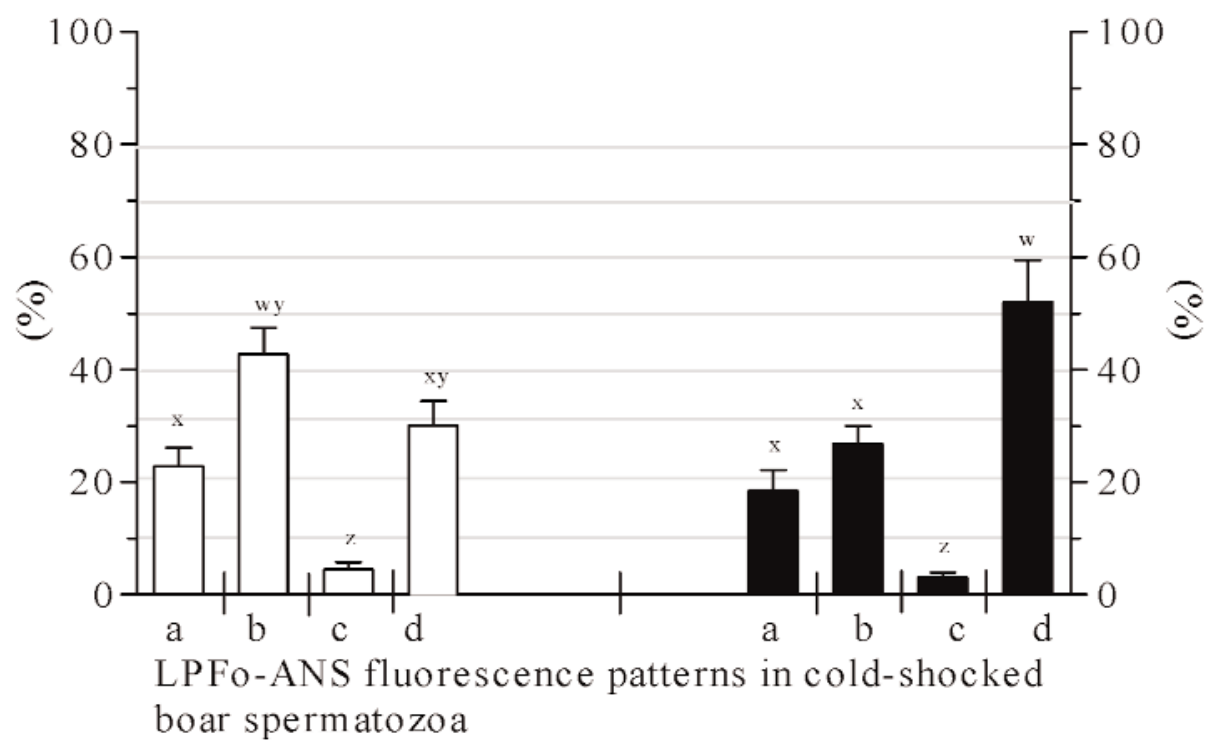

Fig. 3. Percentage of cold-shocked spermatozoa showing LPFo-ANS fluorescence patterns immediately after LPFo treatment $(0 \mathrm{~h})$ and after $3 \mathrm{~h}$ of storage at $16^{\circ} \mathrm{C}(3 \mathrm{~h})$. The $\mathrm{K} 3$ extended semen was supplemented with (A) 2\% LPFo and (B) 5\% LPFo. The degree of LPFo-ANS fluorescence was scored subjectively using the following scoring system for each group of spermatozoa, as described in Fig. 1. Values represent the means $( \pm$ SEM) of 9 ejaculates from 3 boars. Values with different letters $(\mathrm{w}, \mathrm{x}, \mathrm{y}, \mathrm{z})$ are significant at $\mathrm{p}<0.05$.

deterioration in the sperm metabolism activity could have a quenching effect on ANS fluorescence [18]. However, our results showed that there were no marked post-storage deteriorations in sperm metabolism activity, represented mainly by motility and mitochondrial function. It should be emphasized that the interaction of egg yolk components with the sperm membrane is obviously complex. Several mechanisms have been proposed to explain the membrane-associated ANS fluorescence. Studies have demonstrated that the binding of ANS molecules to components of the lipid-protein structures of membrane bilayer is associated with the transfer of excitation energy from the aromatic tryptophan or tyrosine resides bound to the probe [19-20].

In the current study, the fluorescence enhancement accompanying ANS binding to LPFo-coated membranes varied among boars and could be related to dif- ferences in seminal plasma components. Domains of boar sperm membranes have different molecular dynamics and different responses to components of the seminal plasma or cryopreservation media, which could modulate the molecular interactions of egg yolk lipoprotein with spermatozoa [1,21-22]. It is not easy to unravel the role of seminal plasma components on sperm function during liquid storage or cryopreservation of boar semen mainly because of their complex composition [23]. Moreover, the intensity of ANS fluorescence showed that the action of sperm coating by LPFo was more obvious after storage, suggesting that the lipoprotein fraction confers protection to the sperm membrane. It has been postulated that the major protective factor from hen egg yolk is the low-density lipoprotein fraction (LDL), which is largely responsible for protecting spermatozoa during semen preservation [24], probably by the sequestration of detrimental 
Table 1. Sperm plasma membrane integrity (PMI) and mitochondrial function (MF) of boar spermatozoa immediately after LPFo treatment $(0 \mathrm{~h})$ and after $3 \mathrm{~h}$ of storage $(3 \mathrm{~h})$ at $16 \mathrm{C}$ in $\mathrm{K} 3$-extended semen supplemented with $2 \%$ or $5 \%$ LPFo. Values represent the means $( \pm$ SEM) of 3 ejaculates, 3 each from the 3 boars. Within columns, values $(x, y, z)$ with different letter are significant $(\mathrm{p}<0.05)$.

\begin{tabular}{|c|c|c|c|c|c|c|c|c|}
\hline \multirow{3}{*}{ Boar } & \multicolumn{7}{|c|}{ K3-extended semen supplemented with LPFo } \\
\cline { 2 - 9 } & 0 h storage with 2\%LPFo & $3 \mathrm{~h}$ storage with 2\%LPFo & 0 h storage with 5\%LPFo & \multicolumn{3}{|c|}{$3 \mathrm{~h}$ storage with $5 \% \mathrm{LPFo}$} \\
\cline { 2 - 9 } & PMI & $\begin{array}{c}\text { Mitochondrial } \\
\text { function }\end{array}$ & PMI & $\begin{array}{c}\text { Mitochondrial } \\
\text { function }\end{array}$ & PMI & $\begin{array}{c}\text { Mitochondrial } \\
\text { function }\end{array}$ & $\begin{array}{c}\text { PMI } \\
\text { PMitochondrial } \\
\text { function }\end{array}$ \\
\hline F & $80.3 \pm 1.9^{\mathrm{x}}$ & $79.4 \pm 2.5^{\mathrm{x}}$ & $83.5 \pm 3.2^{\mathrm{x}}$ & $83.6 \pm 2.1^{\mathrm{x}}$ & $82.3 \pm 1.5$ & $80.2 \pm 3.5$ & $83.7 \pm 3.1$ & $84.3 \pm 2.1$ \\
\hline $\mathrm{K}$ & $72.5 \pm 1.2^{\mathrm{y}}$ & $70.3 \pm 2.2^{\mathrm{y}}$ & $74.7 \pm 1.1^{\mathrm{y}}$ & $72.7 \pm 2.4^{\mathrm{y}}$ & $74.0 \pm 1.2$ & $76.3 \pm 2.4$ & $76.5 \pm 1.7$ & $77.7 \pm 2.8$ \\
\hline $\mathrm{M}$ & $81.3 \pm 2.2^{\mathrm{x}}$ & $77.3 \pm 1.1^{\mathrm{xy}}$ & $80.4 \pm 2.3^{\mathrm{xy}}$ & $80.7 \pm 3.2^{\mathrm{xy}}$ & $81.2 \pm 3.4$ & $78.5 \pm 1.9$ & $82.7 \pm 2.2$ & $81.3 \pm 2.3$ \\
\hline
\end{tabular}

K3 - Kortowo 3 extender; LPFo - lyophilized lipoprotein fraction of ostrich egg yolk; R123/PI - Rhodamine 123 with propidium iodide.

seminal plasma proteins and binding to spermatozoa [10-11]. Moreover, it has been reported that the phospholipids, particularly phosphatidylcholine, improve membrane fluidity and protect spermatozoa by interacting with their plasma membrane [25]. In the present study, the high proportions of sperm populations defined by LPFo-ANS fluorescence over the head and midpiece regions reinforce the findings of previous studies, indicating that the interaction of spermatozoa with LPFo has an overwhelming protective effect on the sperm membrane architecture during liquid storage or cryopreservation of semen [4-6,8].

In the second experiment ANS fluorescence showed that the overall distribution of LPFo binding sites on the membranes of cold shock-treated spermatozoa was sparse, indicating low affinity for the probe due to irreversible damage to the membrane structures. Surface molecules exposed at the sperm plasma membrane act as sensors integrating environmental information and signaling cell surface events to the cytoplasm and nucleus promoting thereby a cellular response [26]. Exposure of spermatozoa to cold shock temperature prior to LPFo treatment affected the membrane ability to regulate itself and possible other cellular functions. It is conceivable that severely cold shock-treated spermatozoa show extensive damage to their membrane architecture, involving not only the plasma membrane but also the acrosome [2]. Our results are in accordance with those of previous findings, indicating that boar spermatozoa are unable to recover from cold shock-induced damage [27].

Taken together, the results of this study reaffirm that components of LPFo, presumably phospholipids, confer protection to spermatozoa at different domains of the membrane architecture during liquid storage. It is likely that the considerable variations among boars with regards to the protective action of LPFo could affect the sperm susceptibility to cryoinduced damage. Furthermore, it can be suggested that the ANS fluorescence analysis can be used as a laboratory test, where modifications of the sperm membrane can be accurate- ly monitored in studies attempting to optimize the protocol for cryopreservation of boar semen. Further research efforts are been pursued to investigate the dynamic interactions of LPFo components with sperm membrane structures at different stages of the cryopreservation procedure in order to improve the freezability of boar semen.

Acknowledgements: This study was supported by a grant from the National Centre for Research and Development (No.12001404) and funds from Warmia and Mazury University in Olsztyn (0103.0206). A patent application regarding the use of lyophilized lipoprotein fraction of ostrich egg yolk as a protective component of extender for boar semen preservation has been filed at the Patent Office of the Republic of Poland (P-388264).

\section{References}

[ 1] Buhr MM, Curtis EF, Somnapan Kakuda N. Composition and behaviour of head membrane lipids of fresh and cryopreserved boar sperm. Cryobiology. 1994;31:224-238.

[2] Watson PF, Plummer. The response of boar sperm membranes to cold shock and cooling. In: Deep freezing of boar Semen. Edited by L.A. Johnson, K. Larsson, Swedish Univ Agric. Sci. Uppsala, 1985, pp. 113-127.

[3] Buhr MM, Pettitt MJ. Frozen-thawed boar sperm: isolation of membranes and fluidity measurement. Reprod Domest Anim. 1995;31:147-152.

[4] Strzeżek J, Lecewicz L, Dziekońska A, Fraser L. A simple method of extraction of lipoprotein fractions from avian egg yolk - protective effect on cooled boar semen. Theriogenology. 2005;63:496-497.

[ 5] Strzeżek J, Lecewicz M, Fraser L. Ostrich egg yolk as a component of an extender for liquid preservation of boar semen at $5^{\circ}$ and $16^{\circ} \mathrm{C}$. Abstract of the 3rd Ann Conf Europ Soc Domes Anim Reprod (ESDAR), 26-27th November, Anger (France), 1999:86.

[ 6] Dziekońska A, Fraser L, Strzeżek J. Effect of different storage temperatures on the metabolic activity of spermatozoa following liquid storage of boar semen. J Anim Feed Sci. 2009; 18:638-649.

[ 7] Fraser L, Strzeżek J. Effect of different procedures of ejaculate collection, extenders and packages on DNA integrity of boar spermatozoa following freezing-thawing. Anim Reprod Sci. 2007;99:317-329.

[ 8] Strzeżek J, Fraser L, Dziekońska A, Lecewicz M. Complexity of technology preservation of boar semen - fundamental 
and applicative approaches. Reprod Domest Anim. 2006; 41:325.

[ 9] Fraser L, Strzeżek R, Strzeżek J. Fertilizing capacity of boar semen frozen in an extender supplemented with ostrich egg yolk lipoprotein fractions - a preliminary study. Pol J Vet Sci. 2007;10:131-135.

[10] Manjunath P, Veronica N, Bergeron A, Menard M. Major proteins of bovine seminal plasma bind to the low-density lipoprotein fraction of hen's egg yolk. Biol Reprod 2002;67: 1250-1258.

[11] Bergeron A, Manjunath P. New insights towards understanding the mechanisms of sperm protection by egg yolk and milk. Mol Reprod Dev. 2006;73:1338-1344.

[12] Cookson AD, Thomas AN, Foulkes JA. Immunochemical investigation of the interaction of egg yolk interactions with bovine spermatozoa. J Reprod Fert. 1984;70:599-604.

[13] Watson PF. The interaction of egg-yolk and ram spermatozoa studied with a fluorescent probe. J Reprod Fert. 1975;42: 105-111.

[14] Mac Donald BJ, Foulkes JA. A spectrofluometric investigation, using 1-anilino naphtalene-8-sulphonate of the interaction between washed bovine spermatozoa and seminal plasma or egg-yolk lipoprotein. J Reprod Fert. 1981;63:407-414.

[15] Hawe A, Sutter M, Jiskoot W. Extrinsic fluorescent dyes as tools for protein characterization. Pharmal Res. 2008;25: 1488-1499.

[16] Gorgani-Nemat M, Dodd G. The interaction of phospholipid membranes and detergents with glutamate dehydrogenase. Eur J Biochem. 1977;74:139-147.

[17] Garner DL, Johnson LA. Viability assessment of mammalian sperm using SYBR-14 and propidium iodide. Biol Reprod. 1995;53:276-284.

[18] Matulis D, Lovrien R. 1-Anilino-8-naphthalene sulfonate anion-protein binding depends primarily on ion pair formation. Biophy J. 1998;74:422-429.
[19] Mercado E, Rosado A. Structural properties of the membrane of intact human spermatozoa. A study with a fluorescent probes. Biochim Biophys Acta. 1973;298:639-652.

[20] Maddaiah VT, Kumbar U. Membrane permeability transition promoted by phosphate enhances 1-anilino-8-naphthalene sulfonate fluorescence in calcium-loaded liver mitochondria. J Bioenerg Biomembr. 1993;25:419-427.

[21] Strzeżek J, Hopfer E. Zinc ion-dependent protein in boar semen. I. Egg yolk precipitating activity and some biochemical properties. Anim Reprod. 1987;13:117-131.

[22] Glogowski J, Strzeżek J. Studies upon the composition of a diluent for freezing boar semen. I. The dependence of lipoproteins of egg-yolk on seminal plasma proteins. Adv Agric Sci. 1986;263:243-255 (in Polish).

[23] Rath D, Bathgate R, Rodríguez-Martínez H, Roca J, Strzeżek J, Waberski D. Recent advances in boar semen cryopreservation. In: Rodriguez-Martinez H, Vallet JL, Ziecik AJ, eds. Control of Pig Reproduction VIII. Edited by Nottingham University Press, UK: Soc Reprod Fertil. 2009;66:51-66.

[24] Demianowicz W, Strzeżek J. The effect of lipoprotein fraction from egg yolk on some of the biological properties of boar spermatozoa during storage of the semen in liquid state. Reprod Domest Anim. 1996;31:279-280.

[25] Quinn PS, Chow PYW, White IG. Evidence that phospholipids protect spermatozoa against cold shock at a plasma membrane site. J Reprod Fertil.1980;60:403-407.

[26] Calvete JL, Sanz L, Edlin M, Töpfer-Petersen E. Sperm surface proteins. Reprod Domest Anim. 1995;31:101-105.

[27] De Leeuw FE, Colenbrander B, Verkleij AJ. The role membrane damage plays in cold and freezing injury. Reprod Domest Anim. 1991;Suppl. 1:95-104.

Submitted: 16 October, 2010 Accepted after reveiws: 11 March, 2010 